\title{
Niñeces migrantes en el Centro Histórico de Puebla. Estrategias para la integración social
}

\author{
Migrant children in the Historic Center of Puebla. \\ Strategies for social integration
}

\section{Resumen}

Autores:

Adriana Hernández Sánchez* adriana.hernandezsanchez@correo.buap.mx Christian Enrique De La Torre Sánchez*
christian.delatorre@correo.buap.mx

*Benemérita Universidad Autónoma de Puebla

México

Recibido: 09/Ago/2020 Aceptado: $31 /$ Oct/2020
$\mathbf{L}$ a investigación pretende visibilizar la migración mazateca que llega al Centro Histórico de la ciudad de Puebla (México) y que se establece principalmente en vecindades de barrios vulnerables del sector norponiente, como el barrio del Refugio. Son familias numerosas, con más de cinco hijos, que desarrollan su vida en el entorno de una ciudad antigua, que viven en un solo cuarto, dentro de inmuebles deteriorados, que ocupan los patios y los sanitarios colectivos. Se enfrentan a situaciones donde los menores son excluidos por otros niños y adolescentes del barrio debido a su origen, idioma y rasgos físicos. Ante esta problemática, un grupo de profesores y alumnos de la Facultad de Arquitectura (BUAP) y el grupo Re Genera Espacio (RGE), desarrollan estrategias de integración mediante la mejora del espacio público, con acciones que incluyen la construcción de mobiliario, la limpieza y mantenimiento de áreas de uso común, la construcción de un huerto comunitario, realizando actividades lúdicas, como talleres artísticos (dibujo, pintura, maquetas) y funciones de cine.

Palabras clave: infancia; pobreza; barrios; exclusión social; migración.

\section{Abstract:}

This research pretends to make visible the Mazatec migration that arrives to the Historic Center of Puebla (Mexico) and that mainly establish in vulnerable neighborhoods (barrios) of northeast sector, as El Refugio. They are large families with more than five children, who develop their lives in the surroundings of an old city, who live in a single room within deteriorated buildings, which occupy courtyards and collective toilets. They live situations where minors are excluded by other children and adolescents from the neighborhood due to their origin, language and physical characteristics. For abording the problem, a group of professors and students from Faculty of Architecture (BUAP) and Re Genera Espacio group (RGE), develop integration strategies through the improvement of public space, with activities that include the incorporation of furniture to realize various activities, cleaning and maintenance of common use areas, construction of a community garden and realizing recreational activities, as artistic workshops (drawing, painting and modeling) and film shows.

Keywords: childhood; poverty; neighborhoods; social exclusion; migration. 


\section{Introducción}

El reconocimiento de la población indígena migrante que vive en los centros de las ciudades mexicanas no es considerado por las autoridades locales, aunque su presencia sea parte de la vida cotidiana a través del comercio ambulante, la ocupación de vecindades y la asistencia de los niños a las escuelas públicas. Las comunidades indígenas, como la mazateca, son invisibilizadas y vulneradas en la ciudad de Puebla, evidenciando una problemática que las deja indefensas ante los planes gubernamentales que abogan por el repoblamiento del Centro Histórico con nuevos habitantes, y la expulsión de los vecinos con varios años viviendo en el lugar, $y$ también de las familias indígenas que llegan a los barrios. Tampoco existen estrategias para su inclusión.

Es preocupante la situación que viven niñas, niños y adolescentes mazatecos. Aunque la mayoría asisten a la escuela, las posibilidades de deserción son altas debido a situaciones familiares, problemas de aprendizaje derivados de las dificultades en el dominio del idioma español y su pronta incorporación al comercio informal, donde quedan expuestos a intereses de grupos y autoridades que controlan esa actividad. Como otros grupos, enfrentan situaciones de exclusión a nivel material (pobreza y falta de vivienda), social (acceso limitado a la educación y salud), política (ausencia de derechos ciudadanos) y existencial (carencia de expresión pública y capacidades creativas) (Capel, 2016, pp. 257-258).

La región mazateca abarca algunos municipios del norte de Oaxaca y del sur de Puebla y Veracruz, entre las regiones de La Cañada y el valle de Papaloapan-Tuxtepec (Instituto Nacional de los Pueblos Indígenas [INPI], 2017). Desde el siglo XIX, ha padecido diversos procesos de expulsión influenciados por políticas decididas en el centro del país.

Los centros históricos son una construcción social en diferentes ámbitos (Coulomb, 2016, p. 20). Pero en la práctica, se trata de criterios impuestos por la economía internacionalizada en términos de políticas urbanas y fijación de precios, valorización y desvalorización de sectores y actividades, que se han extendido a los centros históricos acreditados por la Unesco, bajo el impulso de sus propias autoridades y que se une a la capacidad de los agentes inmobiliarios y financieros para apropiarse de las plusvalías generadas en el medio urbano, convertido en una mercancía para producir y vender (Capel, 2016, p. 210). Son acciones gestoras y urbanísticas cuyo propósito es liberar los antiguos centros de supuestos factores de devaluación, como "la usurpación por parte de sectores sociales insolventes o problemáticos" (Delgado Ruíz, 2014, p. 199), indignos de la consideración que merecen esos espacios por su valor arquitectónico, histórico o cultural. Así, estos sectores tienen dos lecturas simultáneas: la vulnerabilidad y ser contenedores de tradiciones y saberes.

Vidal, Remesar y Ricart abogan por realizar propuestas encaminadas a la participación ciudadana para la toma de decisiones y acciones específicas en los territorios (2008, p. 4). Atanacio, Sánchez y Hernández mencionan que tener una visión de la ciudad, a partir del diálogo, es una responsabilidad y también un derecho (2014, p. 16)

Vivir una niñez migrante y pobre impacta en las dinámicas escolares, en prácticas y conductas; es una situación influida por la desigualdad y la experiencia en una sociedad fragmentada (Montesinos y Sinisi, 2003 , pp. 68 y 70). A nivel territorial, se manifiesta en la segregación, donde las personas con menores recursos económicos llegan a las periferias de la ciudad; pero también hay casos como los mazatecos, que viven en barrios centrales que no son de vocación turística, donde las rentas son bajas y hay escuelas públicas cercanas para sus hijos. Tonucci menciona que el centro histórico es el lugar donde los niños podrían vivir bien, gracias a toda la infraestructura a nivel espacio público que hay para el desplazamiento y el juego $(2015$, p. 104), pero se requieren priorizar procesos de participación con niños y adolescentes en búsqueda de expectativas de un futuro mejor al que tuvieron sus padres, que requiere tiempo y dedicación, trabajando fuera de los ámbitos escolares, en la casa y en la calle, con los vecinos de toda la vida.

El presente trabajo aborda las acciones realizadas con un grupo de niños del barrio del Refugio, donde el objetivo es establecer una estrategia de participación ciudadana que posibilite la mejora de estos sectores, donde los niños migrantes, mediante la vinculación con universitarios y otros voluntarios, tengan un proceso de integración a la zona adonde llegan, en este caso el Centro Histórico de Puebla.

La metodología planteada es de orden cualitativo y de intervención sobre el sitio a través de procesos participativos, con un equipo interdisciplinar que promueve la gestión de talleres, la realización de mejoras físicas del lugar, estudios sobre el inmueble y sus dinámicas, además de actividades lúdicas en patios y otros espacios públicos. Mediante la renta de un cuarto en la vecindad del Pocito se vincula al equipo con el barrio del Refugio, se desarrollan las actividades por parte de los voluntarios de Re Genera Espacio (RGE) y la Facultad de Arquitectura de la Benemérita Universidad Autónoma de Puebla (FABUAP), mediante la línea de investigación Espacio Público, Participación Ciudadana y Centro Histórico.

\section{Marco Teórico}

Entre 1963 y 1971, en México se implementó una política denominada desarrollo estabilizador que generó un crecimiento de la economía, pero afectó a la agricultura y propició que los campesinos migraran a la ciudad buscando trabajo, coincidiendo con un acelerado proceso de urbanización del país (Cabrera, 2015, p. 
59). Los recién llegados habitaron las vecindades de los barrios; en el primer cuadro de la ciudad se incrementó el deterioro físico de los espacios ante el abandono de los antiguos propietarios y las modificaciones para usos más rentables, como comercios, bodegas y oficinas. El comercio ambulante se desbordó y las actividades delictivas aumentaron con bandas formadas por jóvenes y menores de edad (Hernández, de la Torre, 2014, pp. 54-55). Así, se reforzó un estigma que permanece hasta nuestros días: en los barrios viven los ladrones, pero en el centro ocurren los robos. Como menciona Mejía: "la distribución de delincuentes registrados sigue la geografía de la pobreza y la marginación, pero por otro lado, la actividad delictiva en cambio la geografía de la riqueza" (2013, p. 61). Aunque las autoridades ensalzaban el valor patrimonial de los Centros Históricos, las condiciones de habitabilidad de los barrios eran cada vez más precarias y sin políticas de atención.

Según datos del Instituto Nacional de Geografía y Estadística (INEGI), en el año 2000, en el estado de Puebla se contabilizaron 447,551 personas nacidas en otro estado o país ( $8.8 \%$ de la población total), de las cuales $45 \%$ eran del sexo masculino. De 2010 a 2015, la población indígena de Puebla pasó de 47,667 a 60,989 personas, un incremento del $0.7 \%$ que representaba el $4 \%$ del total de la ciudad (Montagner, 2018). La Encuesta Intercensal 2015 (INEGI), refiere que la migración indígena tiene dos patrones migratorios predominantes: rural-urbano y rural-rural. De acuerdo con información de esa fuente, las principales poblaciones indígenas de México son: nahuas (31\%), mixtecos $(9.7 \%)$, mayas (7.4\%), zapotecos $(6.9 \%)$ y mazatecos $(5.8 \%)$ (Granados y Quezada, 2018, p. 340). ${ }^{1}$

Hablamos de grupos sociales que habitan reducidos espacios de vivienda y utilizan los equipamientos cercanos de los barrios, que encuentran en esta centralidad una posibilidad de vida y una específica geografía de oportunidades (Rasse y Letelier, 2012, p. 147). Son sectores antiguos, con deterioro físico en sus espacios, que conservan dinámicas sociales y los dotan de identidad porque son contenedores de oficios, tradiciones, hábitos, saberes e historias. Es una apropiación constante y dinámica del espacio por parte de quienes lo habitan, no solo un sentido funcional, que da una proyección en el tiempo y garantiza la estabilidad de su propia identidad al ser resumen de vida y de experiencias públicas e íntimas (Pol, 1996, p. 45), en un contexto sociocultural e histórico que "es también de dominio de las significaciones del objeto o del espacio que es apropiado, independientemente de su propiedad legal" (Vidal y Pol, 2005, p. 283). ${ }^{2}$ En los barrios, estas experiencias suceden en calles, parques y en patios de vecindades. Un centro histórico es una realidad arquitectónica y urbana, pero también una construcción social, política, económica y cultural (Coulomb, 2016, p. 20).

A la par de los procesos de exclusión social, hay iniciativas de vecinos, voluntarios y universitarios que pretenden

1 "En lo referente a las principales lenguas indígenas que se hablaban en México, en el año 2015 figuraban como las más relevantes, en orden de importancia: náhuatl, maya, tseltal, mixteco, tsotsil, zapoteco, otomí, totonaco, ch'ol, mazateco, huasteco, mazahua, chinanteco, tarasco, mixe y tlapaneco" (Granados y Quezada, 2018, p. 334) mejorar las condiciones de los habitantes de ciudades históricas. Algunos los definen como una resistencia ante los procesos de expulsión promovidos por el capitalismo, con objetivos y formas de organización basadas en la participación y el trabajo colectivo, "que luchan por conseguir una distribución de bienes y servicios más favorables para la vida cotidiana y para el progreso social, así como para la transformación de la ciudad" (Capel, 2016, p. 258). Así, la participación ciudadana es un factor en los procesos de transformación del espacio público y en la construcción de ciudad y ciudadanía, que brinda herramientas y posibilita canales de comunicación a la comunidad (Vidal, Remesar, Ricart y Raba, 2008, p. 2). Para ello, es necesario reconocer que el espacio público, propiamente público, es "el espacio del conflicto sea este negado o afirmado, latente o patente, contenido o desbocado, resuelto imaginariamente o visibilizado para trabajar políticamente desde el mismo y catalizar una transformación" (Di Masso, Berroeta y Vidal, 2017, p. 83).

\subsection{Estudio de caso: La niñez de la comunidad mazateca en el barrio del Refugio}

Desde hace más de 80 años, en el Centro Histórico de Puebla existe una dinámica migratoria con la región mazateca de Oaxaca que ha sido invisibilizada por autoridades municipales, investigadores y conservadores del patrimonio. Es una situación documentada desde 2014, a partir de una serie de pláticas y entrevistas con vecinos de los barrios del sector norponiente, en Santa Anita, San Antonio y El Refugio (Figura 1). Son ancianos que llegaron a vivir a la zona cuando eran niños, acompañando a sus padres en búsqueda de nuevas oportunidades y se instalaron en las vecindades. Años después, algunos mejoraron su condición laboral y decidieron salir del barrio, mientras que otros se quedaron. A la fecha, siguen llegando de zonas rurales a enfrentarse a ciudades densas $y$, en este caso, a un sector antiguo con problemáticas a nivel urbano, arquitectónico y social. Llegan hablando mazateco, una barrera de acceso a la comunicación, y esto los hace más vulnerables, considerando que los habitantes locales los describen como "personas sin educación que crean problemas".

Aunque el Centro Histórico de Puebla está inscrito en la lista de Patrimonio Cultural de la Humanidad desde 1987, en la práctica las políticas de protección patrimonial han tenido consecuencias a nivel social, resaltando las desigualdades sociales, existentes desde la fundación de la ciudad, entre los núcleos centrales y los barrios periféricos. Esto deriva en condiciones de exclusión social ${ }^{3}$ que acentúan la vulnerabilidad de determinados grupos, como personas de bajos recursos económicos,

\footnotetext{
2 Apropiarse de un lugar no es sólo hacer de él una utilización reconocida sino establecer una relación con él, integrarlo en las propias vivencias, enraizarse y dejar la propia impronta, organizarlo y devenir actor de su transformación." (M. J. Chombart de Lauwe, 1976, p. 524, citado por Pol, 1996, p. 51.)

${ }^{3}$ El fenómeno de la exclusión es cuantificable. Es un indicador para medir el grado de cohesión alcanzado por una sociedad que permite garantizar a personas y grupos desfavorecidos "el acceso a un nive de vida que les permita cubrir sus necesidades materiales básicas y evitarles la marginalidad social" (Consell de Mallorca, 2011, p. 2).
} 


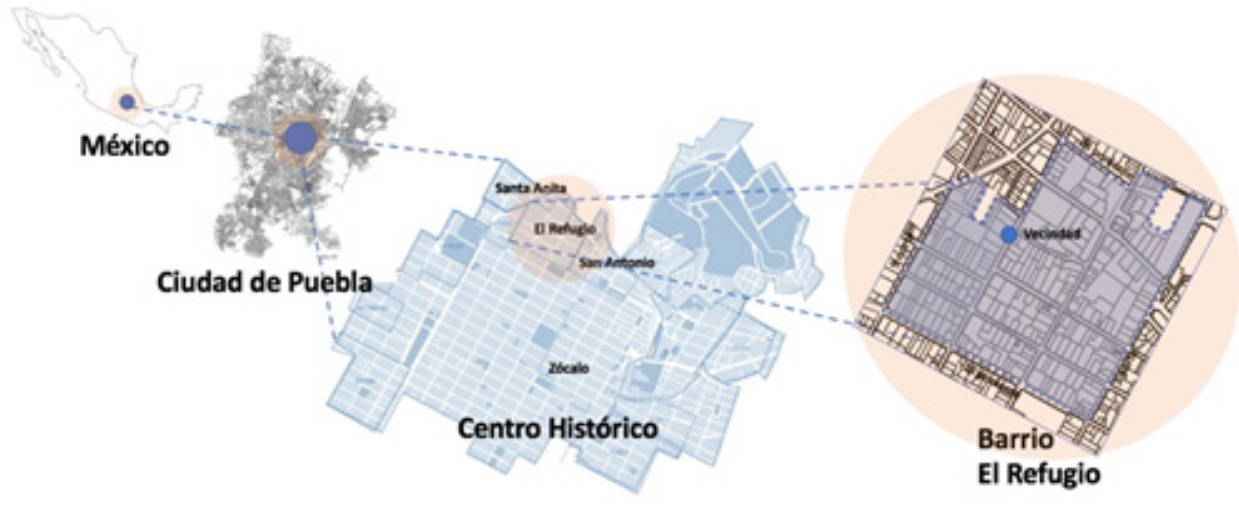

Figura 1: Plano de localización de la ciudad de Puebla, Centro Histórico y Barrio del Refugio Fuente: propia (2020)

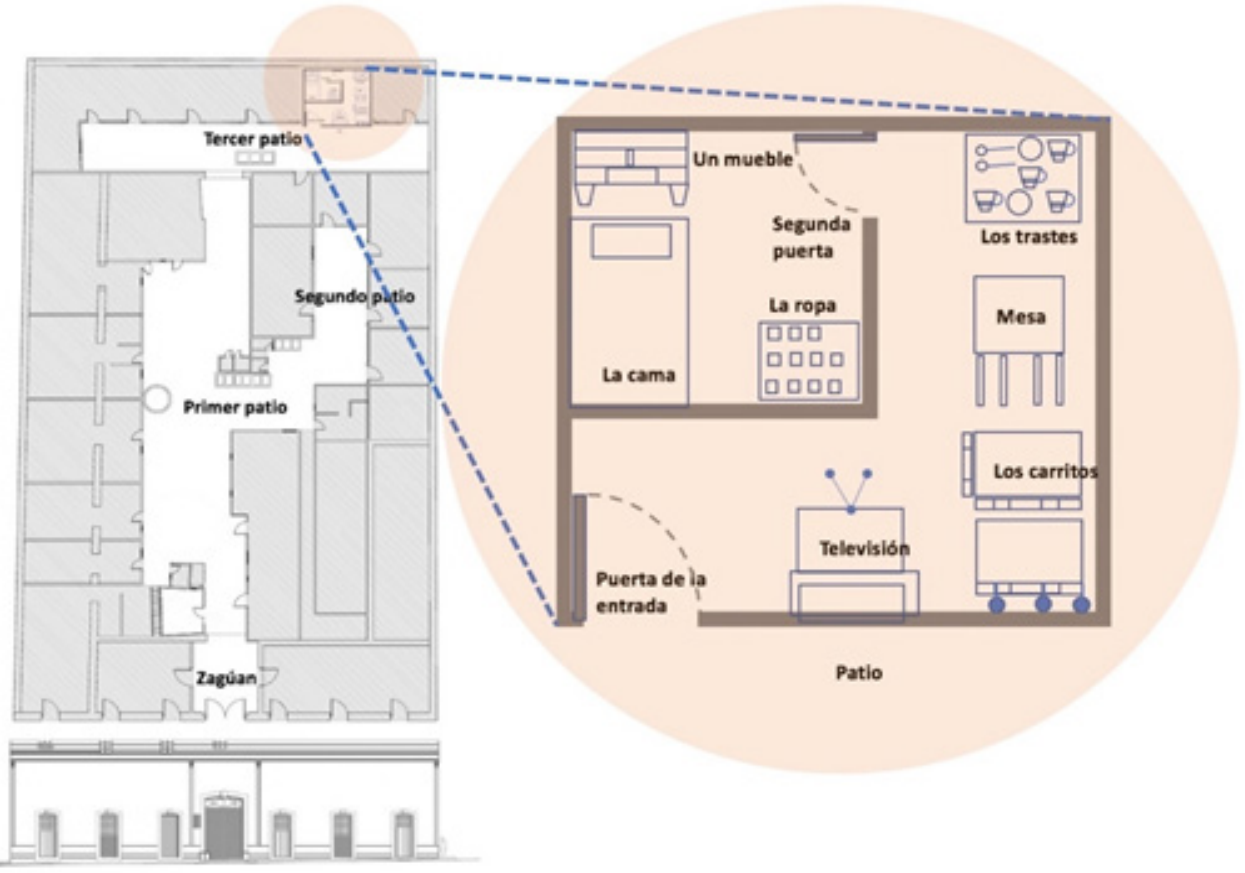

Figura 2: Plano de la Vecindad del Pocito y vivienda familiar conformada por un cuarto, interpretación de "casa" a partir de un dibujo realizado por una niña mazateca de 12 años

Fuente: propia (2020)

ancianos, personas con discapacidad, migrantes, niños y adolescentes, que habitan los espacios con mayor deterioro físico, como las vecindades (Figura 2). ${ }^{4}$

Por otro lado, la vulnerabilidad es también un estado psicosocial que afecta a la percepción que los ciudadanos tienen del territorio en donde viven y de sus propias condiciones sociales. Una percepción negativa de su situación como residentes en un espacio social puede traducirse en procesos de malestar urbano que pueden no corresponderse con unos indicadores 'objetivos'

${ }^{4}$ Algunos autores definen la vulnerabilidad como "aquel proceso de malestar producido por la combinación de múltiples dimensiones de desventaja en el que toda esperanza de movilidad social ascendente, de superación de su condición social de exclusión o próxima a ella es contemplada como extremadamente difícil de alcanzar" (Alguacil, 2006 , p. 161) y conlleva una percepción de inseguridad y miedo a una posible movilidad social descendente, que incluso empeore sus condiciones de vida. En el caso de la vulnerabilidad urbana, se considera que deriva de dos factores principales desencadenantes: la exclusión residencial y la exclusión laboral, que combinadas desencadenan otros factores inhibidores de carácter político, cultural social y personal. (Alguacil, Camacho y Hernández, 2014, p. 78) de vulnerabilidad. Estamos hablando, por tanto, de que la vulnerabilidad es también un concepto relativo, contextual, que debe ser enmarcado en un territorio concreto. (Instituto Juan de Herrera- Departamento de Urbanística y Ordenamiento Territorial, 2010, p. 5)

Son barrios cercanos al primer cuadro de la ciudad, a 14 minutos a pie, a 1.1. kilómetros en línea recta con la principal avenida de la ciudad (Reforma), que comunica a zócalo, el principal espacio público del Centro Histórico. Los migrantes reciben acogida por parte de otros mazatecos ya instalados en El Refugio o en San Antonio, con lazos familiares y de amistad que atraen a nuevas familias. Uno de los beneficios que encuentran al llegar a la ciudad es emplearse como vendedores ambulantes en el sector comercial más importante de la ciudad; llegan caminando al zócalo de la ciudad y acceden a equipamientos cercanos, como mercados, escuelas y espacios públicos. La principal actividad económica es 
la venta de elotes que realizan en la tarde noche por las calles, acompañados de un carrito fabricado por los herreros del barrio. Han ampliado sus actividades a la venta de jugos y otros se dedican a la compra, venta y reparación de carritos eléctricos para niños.

Las vecindades son los inmuebles que mayormente albergan a estas familias. Son espacios que existen desde la época virreinal (siglos XVI-XIX), cuyas habitaciones se organizan alrededor de los patios y conservan una tipología arquitectónica que permite a las familias jóvenes, con cuatro o seis hijos, alquilar uno o dos cuartos que utilizan como vivienda y espacio de trabajo (Figura 3).

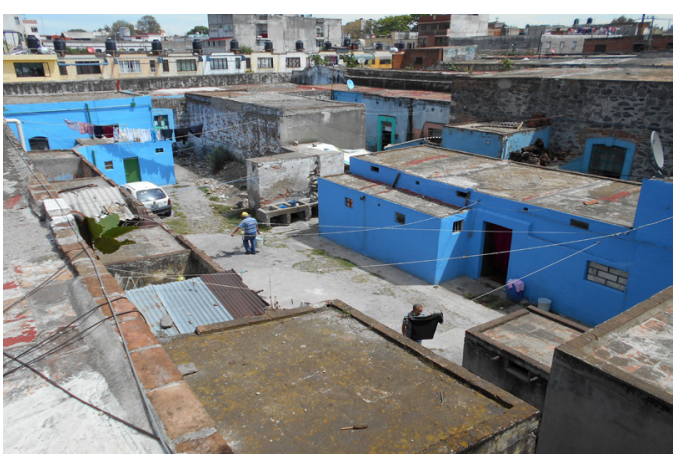

Figura 3: Vista general del patio principal de la vecindad del Pocito, con el módulo de sanitarios y lavaderos colectivos

Fuente: propia (2016)

En 2012, por medio de la línea de investigación se comenzaron actividades en Santa Anita, El Refugio y San Antonio, barrios del sector norponiente de Centro Histórico, para la mejora de espacios públicos a partir de procesos participativos con vecinos. Se han realizado talleres comunitarios, la gestión y elaboración de proyectos con la comunidad. Un año después, el grupo de investigadores y estudiantes dio origen al grupo RGE, conformado por un equipo interdisciplinario (arquitectos, urbanistas, restauradores y conservadores del patrimonio). El objetivo que se persigue es incidir en zonas deterioradas del Centro Histórico de la Ciudad de Puebla que se caracterizan por el deterioro físico de los inmuebles, del espacio público y por la vulnerabilidad social.

El Refugio es un barrio donde una cuarta parte de la población son menores de 14 años (Tabla 1), que vive en una zona histórica con deterioro físico en sus espacios públicos y viviendas. Desde 2013, nuestro principal aliado en la mayoría de estos proyectos ha sido un inquieto grupo de niños, que entonces tenían entre 4 y 10 años, que viven en la calle 24 poniente, conocida como "La Bolsa del Diablo", y continúan trabajando con el equipo de RGE (Figura 4).

En la vecindad del Pocito, las actividades familiares se desarrollan en uno o dos cuartos (de nueve metros cuadrados cada uno) y en dos de los tres patios comunes, el de uso más personal es el que antecede a las habitaciones, donde están los lavaderos y guardan los carros de elotes; mientras que el patio principal, donde hay un pozo de agua, se considera de servicio y algunas

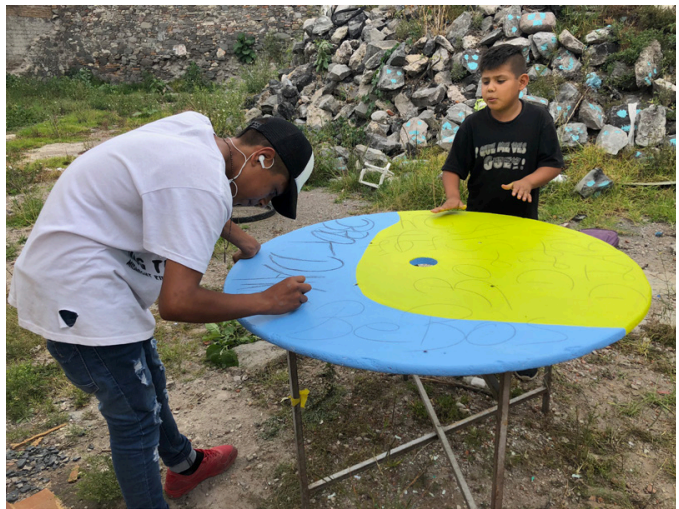

Figura 4: Los niños decorando los muebles de la ludoteca de La Bolsa del Diablo

Fuente: propia (2018)

mañanas los vecinos se levantan temprano para apartar el agua con cubetas.

También ahí se localizan los servicios comunes, como lavaderos y sanitarios, utilizados por niños y adultos.

En los patios se hacen presentes los grupos de niñas y niños mazatecos. La mayoría son hermanos y primos. Desafortunadamente, los demás niños del barrio se burlan de ellos debido a que hablan otro idioma, a las supuestas diferencias en el aspecto físico y la forma de vestir; incluso los imitan en su forma de hablar, tratando de avergonzarlos. Entre los niños mazatecos también hay problemas, sobre todo cuando se trata del juego, y suceden en la diferenciación de las actividades. Mientras que los juegos con la pelota, el salto de cuerda y las escondidas son para todos, las rondas y juegos de manos se consideran actividades femeninas, aunque todos, sin excepción, juegan en el patio principal de la vecindad.

A diferencia de otros niños, los mazatecos no salen solos ni se desplazan fuera del barrio, debido al temor y desconfianza que tienen con algunos vecinos, y solo van a tiendas cercanas a comprar lo que soliciten sus padres. En el caso de las niñas pequeñas, salen con sus hermanas mayores, que toman la responsabilidad de cuidarlas durante largas horas cuando los padres salen a trabajar o realizar compras. A nivel escolar, concluyen la primaria y no contemplan estudiar la secundaria debido a que se incorporan a trabajar, principalmente en el comercio ambulante.

\section{Métodos: La participación de niños en entornos vulnerables}

Los niños del Refugio toda su vida han vivido en el barrio y salen de sus espacios reducidos de vivienda "para vivir con sus amigos las experiencias fundamentales de la exploración, la aventura y el juego" (Tonucci, 2015, p 10). Ocupan los parques cercanos, pero también hacen uso constante de patios de vecindades, banquetas, calles, lotes baldíos y azoteas. En la calle, las formas de agrupación de estos menores hacen que parezcan pandillas, pero en realidad son amigos que tienen cosas 
en común a pesar de la variedad de edades: Juegan fútbol, suben a los techos, juegan canicas, se pelean y reconcilian. Estas interacciones se han ido transformando a través de los años, coincidiendo con la transición de la infancia a la adolescencia. La aparente autonomía en sus desplazamientos diarios en el entorno urbano inmediato es parte de sus condiciones de crecimiento, de relación con la familia y el barrio.

En 2016, identificamos otro grupo de menores que no habían participado en las actividades de años anteriores. Eran muy tímidos, hablaban poco españo y evitaban acercarse a los demás niños. Varios vecinos les llamaban despectivamente "los oaxaquitos". A pesar de las diferencias de los dos grupos, salir a jugar es una necesidad ante los reducidos espacios de las viviendas y la situación laboral de los padres-algunos trabajan largas jornadas o lo hacen en casa-, además de las condiciones de resguardo que les brinda estar cerca de su hogar principalmente en los patios de las vecindades (Figura 5). De ahí la necesidad de plantear una metodología de trabajo para el grupo. Así, se da un acercamiento para desarrollar estrategias de integración entre los niños mazatecos con otros menores del barrio y con las personas adultas, con el espacio público como escenario indispensable para las actividades a realizar.

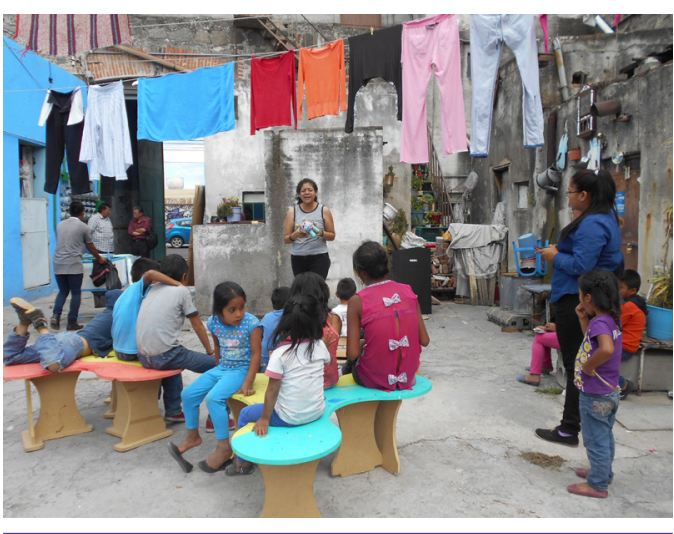

Figura 5: Taller de cuenta cuentos en vecindad del Pocito Fuente: propia (2016)
La metodología (Figura 6) plantea un proceso participativo en diferentes escalas, con acciones inmediatas como parte de una estrategia con perspectiva a largo plazo en búsqueda de una mayor integración de los niños mazatecos con el barrio:

1. Acercamiento con los niños mazatecos a partir de la renta de un cuarto en la vecindad del Pocito, barrio del Refugio, para el desarrollo de diversas actividades de 2016 a la fecha.

2. Incorporación del mobiliario diseñado y construido por los niños de la calle 24 poniente.

3. Conformación de equipos interdisciplinarios de voluntarios, RGE y estudiantes FABUAP, mediante diferentes programas de la Vicerrectoría de Investigación y Estudios de Posgrado BUAP, los Veranos Científicos de la Academia Mexicana de Ciencias y el Consejo Nacional de Ciencia y Tecnología.

4. Gestión y programación de talleristas voluntarios para realizar actividades de educación no formal, periodo 2016-2018, como cursos de dibujo, modelado en plastilina, elaboración de títeres, funciones de cine, cartonería, clases de música y talleres de lectura.

5. Promoción y realización de intervenciones en la vecindad, buscando la mejora de las condiciones de habitabilidad en un marco de conservación del patrimonio: reparación de cuarto en renta, construcción de huerto comunitario, limpieza y mejora de sanitarios públicos (2016-2018).

6. Realización de estudios técnicos en la vecindad Densidad poblacional, características de viviendas, estado de conservación del inmueble.

7. Desarrollo de actividades lúdicas en patios, calles y parques, que ayuden a una relación más respetuosa entre los niños a partir del juego: salto de cuerda, rondas, futbol y otras que conocen los menores.

En los primeros meses, los niños originarios del barrio eran mayoría, mientras que los provenientes de Oaxaca apenas se asomaban a jugar al patio principal. En 2016, a partir de la

Figura 6: Metodología de proceso participativo realizado con niños migrantes mazatecos de la vecindad del Pocito, barrio del Refugio, Centro Histórico de Puebla

Fuente: propia (2020) 
instalación de la sede de RGE en la vecindad, en uno de los cuartos más visibles del patio central, los niños mazatecos comenzaron a salir del tercer patio para acudir a las actividades que se realizaban.

Existe un predominio de las niñas que habitan en El Pocito. Hay tres familias en la vecindad que suman 18 niños, 12 mujeres y 6 hombres, cuyas edades oscilan entre los 2 y 14 años. En el barrio del Refugio, ese rango de edad representa el $26.24 \%$ del total de población (Figura 7 y Tabla 1 ).

Los niños mazatecos acuden a las escuelas primarias oficiales, con todas las problemáticas de integración que conlleva derivadas del desconocimiento, parcial o total,

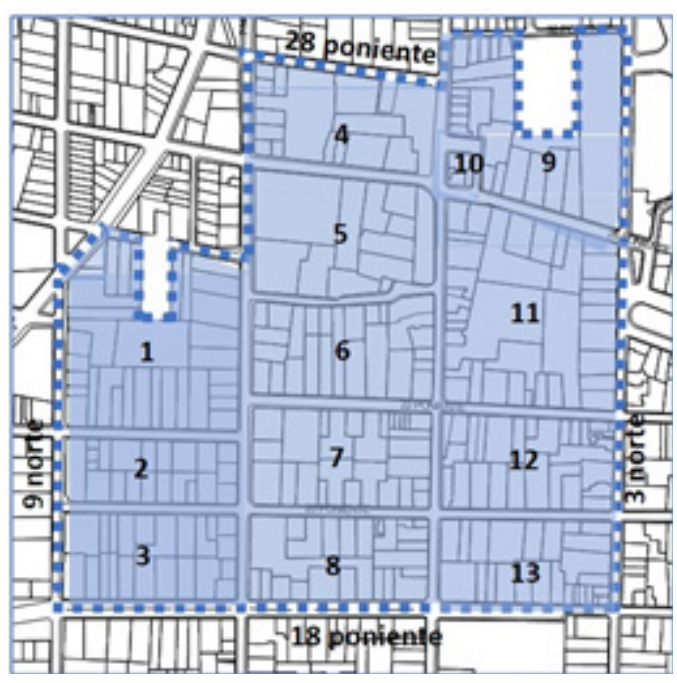

Figura 7: Plano manzanero del barrio del Refugio Fuente: propia (2020) del idioma español y la adaptación al sistema escolar. Las niñas tienen disponibilidad para salir a jugar en grupo al patio, pero tienen que pedir permiso para realizar alguna actividad en la calle. Incluso en algunas ocasiones han solicitado al equipo de voluntarios que pidan permiso a sus padres para acudir a las actividades fuera de la vecindad. Es evidente que tiene más posibilidades de salir a la calle, a jugar sin la compañía de un adulto, un niño que nació en el barrio que una niña que llegó al barrio en años recientes.

El cuarto rentado en la vecindad fue habilitado como taller artístico (dibujo, pintura, escultura, música), otras veces como sala de lectura o cine (Figura 8). También se realizaron jornadas de limpieza de azoteas y patios, y se construyeron un huerto y un jardín vertical (Figura 9), que permanecen hasta la fecha, con algunas modificaciones, y siguen siendo utilizados por los menores y sus padres.

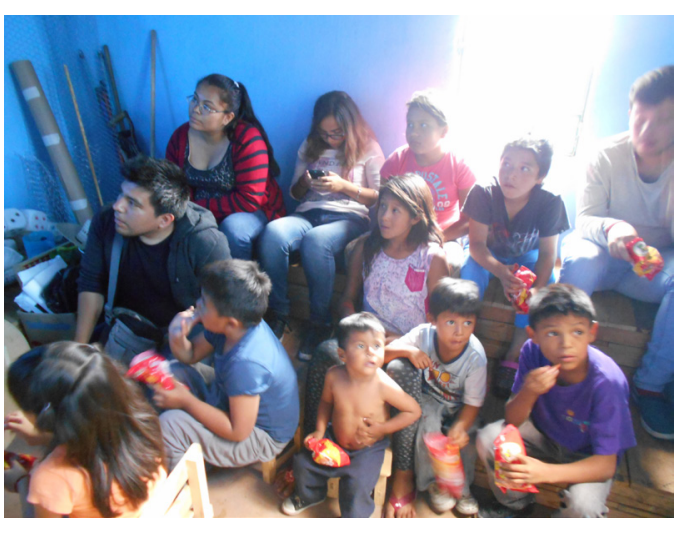

Figura 8: Función del cine en el cuarto de la vecindad Fuente: propia (2016)

\begin{tabular}{|c|c|c|c|c|c|c|}
\hline Manzana & $\begin{array}{c}\text { Población total } \\
\text { (habitantes) }\end{array}$ & $\begin{array}{c}\text { Población de } \\
\text { 0 a 14 años } \\
\text { (habitantes) }\end{array}$ & $\begin{array}{c}\text { Población de } \\
\text { 0 a 14 años } \\
\text { (porcentaje) }\end{array}$ & $\begin{array}{c}\text { Promedio de es- } \\
\text { colaridad (años } \\
\text { escolares) }\end{array}$ & $\begin{array}{c}\text { Total de vi- } \\
\text { viendas (can- } \\
\text { tidad) }\end{array}$ & $\begin{array}{c}\text { Total de vivien- } \\
\text { das habitadas } \\
\text { (cantidad) }\end{array}$ \\
\hline$(1)^{*}$ & 676 & 174 & 25.7 & 10.9 & 289 & 196 \\
\hline$(2)$ & 156 & 33 & 21.1 & 9.3 & 56 & 40 \\
\hline$(3)$ & 81 & 17 & 21.0 & 11.9 & 34 & 27 \\
\hline$(4)$ & 67 & 15 & 22.4 & 12.1 & 21 & 19 \\
\hline$(5)$ & 112 & 26 & 23.2 & 8.4 & 42 & 32 \\
\hline$(6)$ & 436 & 151 & 34.6 & 7.9 & 152 & 112 \\
\hline$(7)$ & 251 & 67 & 26.7 & 9.3 & 94 & 70 \\
\hline$(8)$ & 206 & 53 & 25.7 & 8.3 & 96 & 64 \\
\hline$(9)$ & 162 & 46 & 28.4 & 9.4 & 75 & 55 \\
\hline$(10)$ & 27 & 8 & 29.6 & 10.5 & 8 & 7 \\
\hline$(11)$ & 381 & 108 & 28.4 & 8.8 & 120 & 92 \\
\hline$(12)$ & 247 & 71 & 28.7 & 8.4 & 94 & 75 \\
\hline$(13)$ & 211 & 54 & 25.6 & 9.4 & 77 & 62 \\
\hline Total & 3013 & 823 & 26.54 & 9.6 & 1158 & 851 \\
\hline
\end{tabular}

*Manzana donde se localiza la vecindad del Pocito

Tabla 1: Cantidad de población, menores de edad (0-14 años) y viviendas por manzana en el barrio del Refugio Fuente: propia (2020) a partir de información del Censo General de Población y Vivienda 2010 


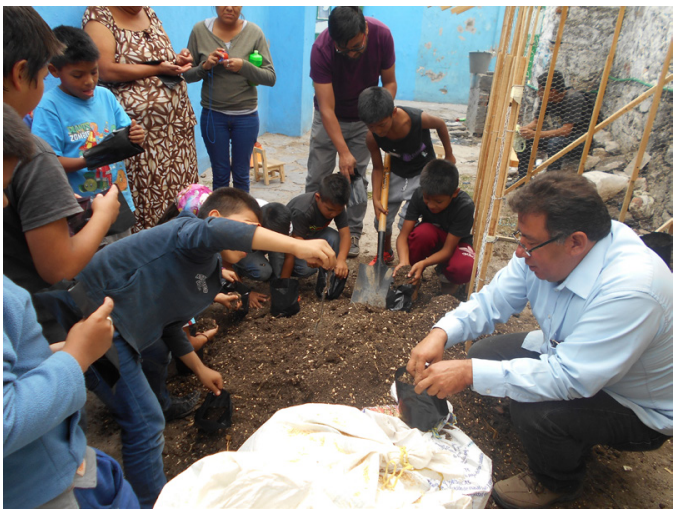

Figura 9: Taller de elaboración de composta Fuente: propia (2016)

En 2017, Ilegaron más familias buscando oportunidades de trabajo y era notoria la ocupación intensa de los patios con triciclos de carga para la venta de elotes. La situación se revertía, los niños oaxaqueños ahora eran mayoría, jugaban en dos de los tres patios, incluyendo el principal, y era común escuchar conversaciones en mazateco, lo que resultó una estrategia defensiva de los menores ante la burla de otros niños y algunos mayores de edad. En lo que respecta a los talleres artísticos, continuaron con una frecuencia de dos veces al mes, incorporándose nuevos voluntarios y actividades, como jornadas de lectura en mazateco y un curso de alfabetización básica para los menores que no acudían a la escuela.

Pero el sismo del 19 de septiembre de 2017 provocó graves daños a una considerable cantidad de edificios antiguos en el centro de la ciudad y sus barrios. En la vecindad del Pocito resultaron dañadas la mayoría de las viviendas y algunas familias decidieron abandonar el lugar. En el caso de los mazatecos, algunos regresaron a sus pueblos de origen, otros se reubicaron en vecindades menos dañadas, en El Refugio y San Antonio. Los dueños de la vecindad repararon pausadamente los cuartos: saben que la opción más viable de renta está con las familias que llevan muchos años en el barrio y con los mazatecos, que pagan rentas económicas.

En el verano de 2018, de manera conjunta entre vecinos, universitarios y voluntarios, se intervinieron los baños comunitarios de la vecindad. Se repusieron aplanados en los muros y se pintaron, como parte de un taller de uso de la cal en la construcción (Figura 10). Para el cumplimiento de las actividades, bajo asesoría y supervisión de especialistas, se iban intercalando los roles, sin importar las edades y género. De esa manera, tanto niños del barrio como jóvenes voluntarios debían realizar el cernido de arena, la preparación de cal, la cuantificación de proporciones, la mezcla de materiales y la aplicación

Durante 2019 y 2020 se continuaron realizando actividades en la vecindad, incorporándose nuevos voluntarios que realizaron talleres de pintura, escultura y arqueología. Cabe mencionar que en los talleres y juegos es habitual la participación de los menores sin diferenciación de roles de género. Incluso en un taller que se denominó “Casa de Muñecas", los más entusiastas

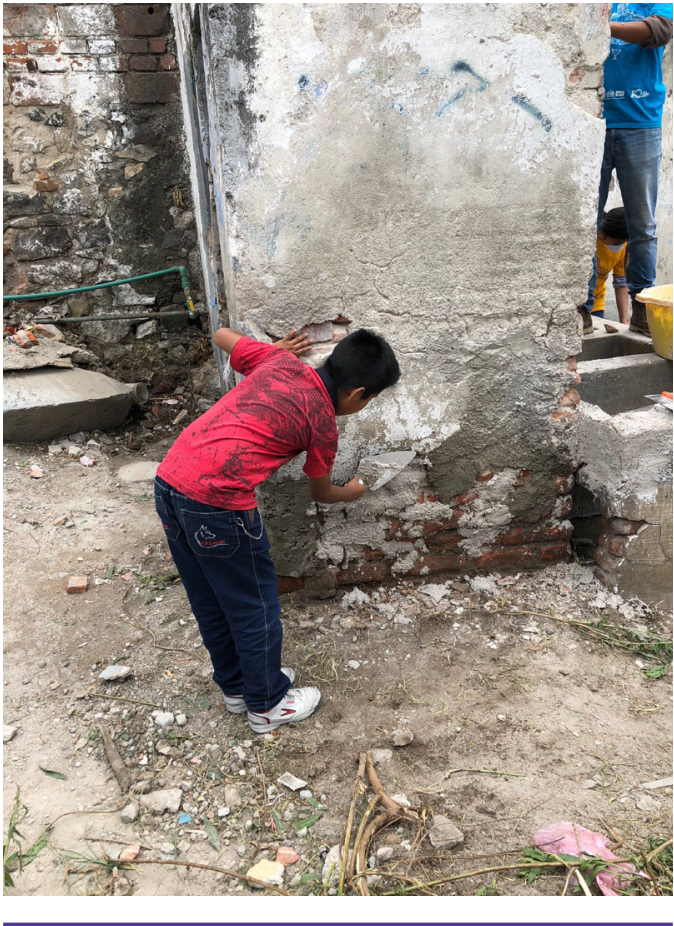

Figura 10: Niño realizando labores de albañilería en muro de baños colectivos

Fuente: propia (2018)

fueron los niños, que inicialmente acudieron a la actividad "para ayudar a sus hermanas menores". Los papeles de colores, el pegamento y los lápices son instrumentos creativos recurrentes en todas las actividades, donde siguen las indicaciones de los facilitadores (Figura 11).

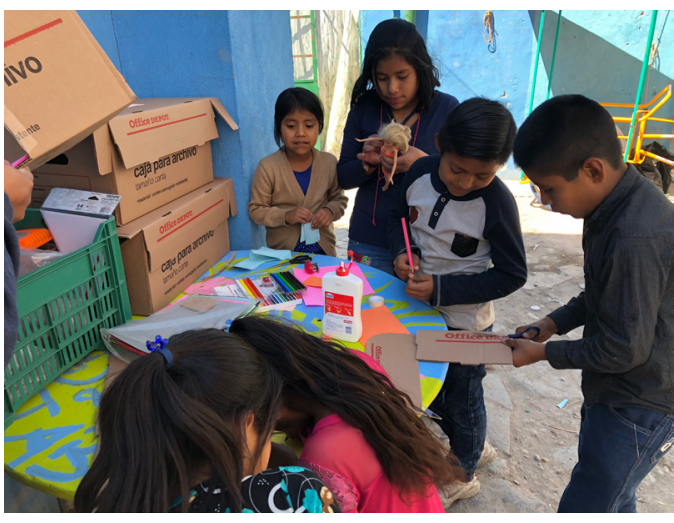

Figura 11: Taller "Casa de Muñecas" Fuente: propia (2019)

En abril de 2020 se suspendieron las actividades debido a la cuarentena impuesta por las autoridades ante la propagación del Covid-19. Las familias mazatecas han tenido problemas económicos severos debido a que ha disminuido la venta de sus productos ante la imposibilidad de salir a la calle, por lo que, como sucedió en septiembre de 2017, algunas decidieron regresar a sus pueblos en espera de que mejore la situación. 


\section{Resultados: actividades con los niños mazatecos de la vecindad del Pocito}

Durante cuatro años las actividades han variado y permitido la integración de niños mazatecos de diferentes edades. Actualmente, son más receptivos y participativos desde una percepción cualitativa, y tienen una mayor comunicación con el equipo. Promueven las actividades, gestionan fechas y horarios, se sienten con mayor confianza, aunque lleguen otros niños del barrio. Además, resguardan el cuarto y los materiales de trabajo y han ayudado en el mantenimiento del mobiliario (Figura 12).

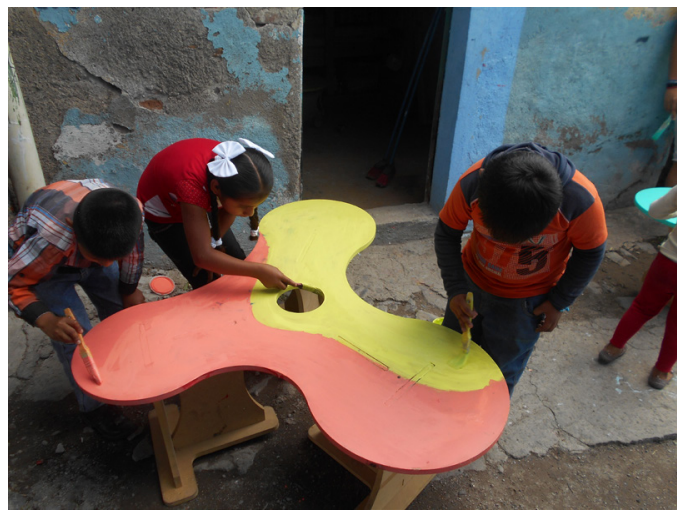

Figura 12: Los niños pintando el mobiliario de los talleres Fuente: propia (2019)

El juego es un elemento cohesionador entre los niños mazatecos, así como con otros menores de la vecindad y los que viven en la 24 poniente. Como grupo, la línea de investigación y RGE, plantean como principal resultado la integración con los menores, en un aprendizaje recíproco que permite visualizar nuevas estrategias, tanto con los menores que hemos trabajado, en su paso a la adolescencia, como con sus hermanos menores. Dando continuidad al trabajo que se realiza en El Refugio, los estudios sobre las viviendas colectivas nos permiten plantear nuevas iniciativas en los barrios, los inmuebles y los espacios públicos, incluyendo los patios de las vecindades, característicos de barrios y colonias antiguas.

El patio tiene la cualidad de ser un espacio público dentro de lo privado, es elemento de articulación con el resto de la ciudad, tiene conexión con otros espacios importantes como la calle y genera una continuidad con el parque. Es flexible y multifuncional, permite el juego, deporte, entretenimiento, educación, trabajo, descanso e intimidad. En las actividades realizadas, las características del patio no restringen las actividades lúdicas por género: niñas y niños participan por igual en el juego de pelota, e salto de cuerda y en talleres como "Casa de muñecas". Es a través del juego que se da esta igualdad de condiciones donde pueden explorar actividades que, habitualmente, se consideran diferenciadas para mujeres y hombres.

Para los niños mazatecos el patio se convierte en un espacio seguro. Es el primer elemento que tienen como preámbulo para conocer el barrio y la ciudad adonde llegan, además de los trayectos hacia las escuelas y otros servicios cercanos que usan, con los aspectos positivos y negativos que pueda haber en esa primera aproximación, pero que poco a poco se apropian principalmente a través de las actividades lúdicas, por lo que resulta positivo que este tipo de viviendas colectivas cuenten con espacios abiertos considerando que los espacios habitables privados tienen dimensiones reducidas. Se requieren promover nuevas investigaciones sobre los espacios colectivos de las viviendas para identificar las diversas maneras de apropiación por parte de los habitantes.

\section{Discusión}

Ante la preocupación de algunos autores respecto a que ciertos enfoques teóricos "no muestran una posición y concepción epistemológica de la intervención de los niños que explicite la naturaleza de la participación infantil" (Espinar, 2003, p. 13), otras investigaciones concluyen que, aunque las niñas y niños tienen capacidades para participar, en la práctica no existen oportunidades para que lo hagan mientras los adultos no cambien su relación con los menores en la manera de reconocerlos y tenerlos en cuenta (Gallego-Henao, 2015, p. 160)

Es urgente entonces que se inicien procesos de educación en este tema, debido a que, si socialmente no se cambia e imaginario que indica que las personas menores no tienen capacidades para participar, entonces los familiares, la sociedad y los agentes educativos seguirán teniendo una mirada futurista $y$ reduccionista de la infancia (Gallego-Henao, 2015, p. 160).

Una posibilidad de construir esa participación con los niños está en los proyectos educativos para la mejora de los espacios públicos, como ha sucedido con los niños del barrio del Refugio. Se trata de que los niños tengan elementos para hacer un uso más consciente de los espacios de uso común, que sepan su valor, generen propuestas y realicen acciones para su mejoramiento:

1. Valorizar el espacio público de manera más amplia e incluyente, que no solo es un espacio de juego.

2. Mejorarlo a través de propuestas y acciones basadas en la participación e inclusión.

3. Valorarlo, con la posibilidad de construir un mejor futuro y entorno, comparado con el que crecieron sus padres.

4. Valorizar las actividades expresivas que realizan y los hacen sentirse integrados e identificados.

En años recientes se ha discutido si "la magnitud de esta inequidad nos habla de niños que, más que "vulnerables", son vulnerados gravemente en su derecho a desarrollar todas sus capacidades humanas":

Hablar de "niños con vulnerabilidad" nos conecta con una emocionalidad de compasión... En cambio, hablar de niños que deben enfrentar adversidades, ello nos conecta con otro tipo de compasión: esa que uno siente con el héroe que "no la ha tenido fácil” (Gaete, 2018)

En este proceso, la participación infantil contribuye al reconocimiento de la diversidad social que ha caracterizado a las ciudades antiguas. No hay que idealizar el concepto de "repoblamiento" con la llegada de nuevos habitantes que desplacen a los vecinos originarios de los barrios ni a los migrantes indígenas. Se deben revalorizar las cualidades que otorgan los espacios 
públicos, y semipúblicos, a los niños y adolescentes en su crecimiento y expectativa de vida. Como menciona Tonucci:

El centro histórico de la ciudad es un lugar donde los niños podrían vivir bien, gracias a las zonas peatonales, gracias a las plazas y a las plazoletas, los jardines y monumentos, las fuentes y la misma estructura urbana, que se presta perfectamente para el desplazamiento y el juego (2015, p. 104).

La concepción epistemológica que "explicite la naturaleza de la participación infantil" ha tenido grandes avances, pero continúa en construcción (Espinar, 2013 p. 13). Hablamos de "diversas niñeces", con relación a "las multiplicidades de maneras de vivir una etapa determinada de la vida, y entre estas maneras sigue existiendo la desigualdad, la subalternidad incrustada en un mundo de discursos correctos y democracias duraderas" (Shabel, 2014, p. 164). El reconocimiento de la diversidad y el papel activo que deben tener los menores de edad, como sujetos de derecho, no solo es una fase previa a la obtención de ciudadanía legal, fuera de la limitada percepción del adultocentrismo. Como menciona Rabello, "la infancia es devenir, volverse, no aquello que ya se sabe que es, o que fue, repitiendo la historia, sino "librándose de ella", de su juego que petrifica las posibilidades del presente" (2001, pp. 4849).

La participación infantil y juvenil se considera un ámbito experimental en el diseño de espacios, con suficientes riesgos de caer en un "marketing" que solo justifique "incorporar" aportaciones de los niños; algunas claves pueden ser "la aproximación creativa, los tiempos adecuados, la observación y una sólida base pedagógica" (Navarro, 2019). ${ }^{5}$ Santiago Atrio, en una entrevista reciente, menciona que "lo importante no es hablar de espacios, es hablar de metodologías, de cómo quieren ustedes que los chicos aprendan" (Osa Menor, 2020 $21 \mathrm{~m} 35 \mathrm{~s})$. A través de procesos participativos basados en proyectos educativos, los niños pueden mejorar sus espacios públicos, como el patio y la calle, incluso con la posibilidad de vislumbrar más expectativas de futuro, o al menos superar las que tuvieron sus padres y abuelos (Figura 13).

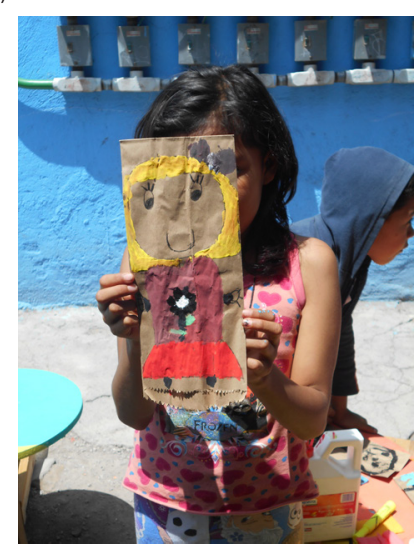

Figura 13: Taller de elaboración de títeres

Fuente: propia (2016)

5 Navarro (2019) menciona que la participación infantil posee características particulares: 1) Está íntimamente ligada a la educación 2) Requiere de métodos indirectos, 3) Niñas y niños deben integrarse en el proceso como especialistas en cuanto a sus necesidades de confort, sociales y de juego, 4) La escala del espacio a intervenir y 5) El tiempo necesario para desarrollar el proceso.
La transición de la infancia a la adolescencia también se debe considerar, en particular con la relación del uso de los espacios públicos y la percepción que los adultos tienen al respecto:

...se considera que los niños más pequeños, en particular, necesitan protección contra el peligro de extraños y los accidentes de tráfico, mientras que los niños mayores se conciben con mayor frecuencia como un riesgo para la tranquilidad y control del espacio público (Holloway, 2014, p. 381)

Un mejor reconocimiento disciplinar de otras áreas de estudio, como la Geografía de los niños, que investiga sus lugares y espacios de vida-en su carácter de experiencia, estética, política, moral y ética-, entre otros aspectos, permitirá un mejor reconocimiento de cómo los niños crean y rehacen el espacio y, por lo tanto, de ellos mismos, y cómo nosotros, como adultos, los habilitamos o restringimos en el proceso de hacer el mundo de nuevo (Aitken, 2018, pp. 18-19)

\section{Conclusiones}

Los niños mazatecos se protegen entre ellos, tienen un idioma en común y una serie de antecedentes que los hace un grupo compacto. Son vulnerados en el barrio, la escuela y la ciudad por ser migrantes y pertenecer a familias humildes.

El juego es importante para ellos, a pesar de que tienen responsabilidades en el hogar y trabajo. Cambian al rol de adultos cuando los mayores les encargan una tarea: cuidar a los hermanos pequeños, bañarlos, lavar trastes y ropa, limpiar los sanitarios colectivos, servir como intérpretes para gestionar alguna ayuda y apoyar en el trabajo. A pesar de su contribución en el ingreso y el cuidado del hogar, habitualmente sus opiniones no son tomadas en cuenta, por lo que también padecen exclusión a nivel familiar. Ante estas situaciones, se debe hablar de distintos tipos de niñeces en los barrios, considerando que no hay un tipo que corresponda a una visión idealizada o habitual de la infancia, adultocentrista, que los considera como receptores de cuidados por parte de los padres y con la única responsabilidad de estudiar.

Los niños se adaptan a las condiciones de los lugares que habitan. Los espacios abiertos, como el patio, se convierten en áreas de juego y no es necesario salir a los parques. Es un escenario que corresponde a la percepción de inseguridad que se vive en el país, pero también se debe a la desconfianza hacia algunas personas que viven fuera de la vecindad, incluyendo otros niños y jóvenes, a pesar de que llevan varios años viviendo en la zona.

Con las actividades realizadas por el grupo RGE y otros voluntarios, se comprueba que los niños se adaptan a las condiciones, y que tienen un sentido de responsabilidad y cuidado cuando les hemos prestado algunos muebles y los devuelven. También está presente la expectativa de los niños con los universitarios respecto a la periodicidad de las actividades: ¿Cuándo vienen los nuevos estudiantes de verano? ¿De dónde son? ¿Qué haremos el Día del Niño? ¿Vamos a tener posada en Navidad? Ante una tendencia generalizada donde parece que la infancia se desvanece por la premura de crecer pronto, influenciada por el mercantilismo y los medios de comunicación, en los menores del Pocito y El Refugio sigue presente esa 
expectativa de seguir siendo niños, de jugar, de convivir y divertirse, a pesar de lo difícil que parezca el entorno.

La metodología se fue transformando según las necesidades de los niños, pero también según los recursos disponibles y las oportunidades que el equipo fue descubriendo. El trabajo realizado es una muestra pequeña de las condiciones sobre cómo los niños migrantes se insertan en nuevos contextos. Estas situaciones, aparentemente atípicas, abren un panorama hacia el reconocimiento de la población indígena, que seguirá llegando a los centros de las ciudades antiguas. En el caso de los mazatecos, es una migración invisibilizada, que lleva sucediendo, según sus palabras, al menos ochenta años, por lo que se requieren acciones por parte de instancias gubernamentales y otros voluntarios para el desarrollo de más actividades enfocadas a los menores y de creación de nuevos equipamientos para los recién llegados.

Al llegar al barrio, los niños no hablaban español, pero a paso del tiempo los hemos escuchado hablar fluidamente y también conservar su idioma. Es necesario crear centros de apoyo para niños migrantes, como parte de su adaptación a las actividades que realizan en la ciudad y en la escuela. Esto les permitiría conservar el mazateco y a la vez mejorar su escritura, incluso con la posibilidad de apoyar en la conservación de su cultura.

Las niñas y niños valoran el trabajo realizado con ellos y respetan a los profesores, como nos llaman indistintamente a alumnos e investigadores. Sabemos que es un esfuerzo focalizado en un grupo pequeño de un sector de la ciudad, pero es muy probable que existan muchos más grupos en otros barrios y colonias, invisibilizados y sin apoyo. Reconocer las niñeces migrantes evidencia el desconocimiento de los centros históricos y su diversidad por parte de investigadores y autoridades, en particular de las administraciones locales que cada tres años están enfocadas en solucionar problemas turísticos y comerciales.

\section{Agradecimientos}

El trabajo realizado con los niños del barrio del Refugio forma parte de las actividades de la línea de investigación "Espacio Público, Participación Ciudadana y Centro Histórico", que coordina Hernández Sánchez, y que dio origen al grupo RGE. Desde 2012, en los meses de junio, julio y agosto se incorporan estudiantes de licenciatura de diversas áreas profesionales y regiones de país como parte del Verano Científico, promovidos por los programas Delfín y Jaguar (CONACYT) y de la Academia Mexicana de Ciencias. En periodos escolares, han participado estudiantes del programa Jóvenes Investigadores de la Vicerrectoría de Docencia de la BUAP. En 2016, De La Torre Sánchez obtuvo la beca del posgrado de excelencia CONACYT para cursar el Doctorado en Procesos Territoriales de la BUAP, lo que permitió la disponibilidad de tiempo para realizar su proyecto de tesis con los niños del barrio. A partir de 2018, también se han incorporado jóvenes voluntarios, locales y extranjeros, que participan en el programa de Voluntarios del Patrimonio Mundial de la Unesco (WHV, por sus siglas en inglés), o que se incorporan por decisión propia, considerando el interés en el tema.
Cómo citar este artículo/How to cite this article: Hernández Sánchez, A. y De La Torre Sánchez, C. (2021). Niñeces migrantes en el Centro Histórico de Puebla. Estrategias para la integración social. Estoa. Revista de la Facultad de Arquitectura y Urbanismo de la Universidad de Cuenca, 10(19), 77-88. doi: 10.18537/est.v010.n019.a07

\section{Referencias bibliográficas}

Alguacil, J. (2006). Barrios desfavorecidos: diagnóstico de la situación española. En F. Vidal (Ed.), V Informe FUHEM de políticas sociales: La exclusión social y el estado del bienestar en España (pp. 155-168). FUHEM.

Alguacil, J., Camacho, J. y Hernández, A. (2014). La vulnerabilidad urbana en España. Identificación y evolución de los barrios vulnerables. Empiria. Revista de Metodología de Ciencias Sociales, (27),73-94.

Aitken, S. (2018). Children's Geographies: Tracing the evolution and involution of a concept. Geographical Review, 108(1), 3-23. http://doi:10.1111/gere.12289

Atanacio, I., Sánchez, J. y Hernández, A. (2014). PIUS, Programas e Iniciativas Urbano Sociales. Estrategias participativas para construir ciudades. Universidad Iberoamericana Puebla y Benemérita Universidad Autónoma de Puebla.

Cabrera, V. (2015). Elitismo de la política urbana. Centro Histórico de Puebla, México. Mañongo, XXIII (44), 43-70.

Capel, H. (2016). Violencia, inequidad, contaminación y otros problemas en la ciudad actual ¿Qué hacer?. En N. Benach y A. Alessandrini (Eds.), Horacio Capel. Pensar la ciudad en tiempos de crisis (pp. 225-270). Icaria.

Consell de Mallorca. (2011). Pla Sectorial per a la Inclusió Social de Mallorca 2011-2014. Consell de Mallorca.

Coulomb, R. (2016). Reestructuración económica, patrimonio histórico y turismo cultural en la planeación urbana de los centros históricos de México. En G. Milián, M. Flores y B. Téllez (Eds.), Complejidad e cincertidumbre? Un nuevo aliento para la ciudad histórica (pp. 19-40). Benemérita Universidad Autónoma de Puebla.

De La Torre-Sánchez, C. y Hernández Sánchez, A. (2014). El Paisaje. En A. Hernández Sánchez (Ed.), Barrio Rojo San Antonio (pp. 21-60). Benemérita Universidad Autónoma de Puebla.

Delgado, M. (2014). La memoria insolente. Luchas sociales. en centros históricos. En L. Durán, E. Kingman y M. Lacarrieu (Eds.), Habitar el Patrimonio. Nuevos aportes al debate desde América Latina (pp. 196-209). FLACSO.

Di Masso, A., Berroeta, H. y Vidal, T. (2017). El espacio público en conflicto: Coordenadas conceptuales y tensiones ideológicas. Athenea Digital, 17(3), 53-92. https://doi.org/10.5565/rev/athenea.1725 
Espinar, Á. (2003). "El ejercicio del poder compartido". Estudio para la elaboración de indicadores e instrumentos para analizar el componente de participación de niños y niñas en proyectos sociales. Escuela para el DesarrolloSave the Children Suecia.

Gaete, J. (25 de septiembre de 2018). ¿Niños vulnerables o vulnerados? Noticias UAl. https://noticias.uai.cl/ columna/ninos-vulnerables-vulnerados/

Gallego-Henao, A. (2015). Participación infantil... Historia de una relación de invisibilidad. Revista Latinoamericana de Ciencias Sociales, 13(1), 151-165. http://dx.doi. org/10.11600/1692715x.1318060514

Granados, A. y Quezada, M. (2018). Tendencias de la migración interna de la población indígena en México, 1990-2015. Estudios Demográficos y Urbanos, 33 (2), 327-363.

Holloway, S. (2014). Changing children's geographies. Children's Geographies, 12(4), 377-392. https://doi.org/ 10.1080/14733285.2014.930414

Instituto Juan de Herrera- Departamento de Urbanística y Ordenamiento Territorial. (2010). Análisis urbanístico de Barrios Vulnerables en España. Sobre la Vulnerabilidad Urbana. Ministerio de Fomento del Gobierno de España.

Instituto Nacional de Estadística y Geografía. (2016) Inventario Nacional de Viviendas 2016. https://www. inegi.org.mx/app/mapa/inv/

Instituto Nacional de los Pueblos Indígenas. (20 de octubre de 2017). Etnografía del pueblo mazateco de Oaxaca - Ha shuta Enima. https://www.gob.mx/inpi/ articulos/etnografia-del-pueblo-mazateco-de-oaxaca-hashuta-enima

Mejía, C. (2013). Valoración de la cultura ciudadana en el espacio público posterior a una renovación urbana. Caso de estudio barrio El Carmel [Tesis de Máster, Universitat Politècnica de Catalunya]. https://upcommons.upc.edu/ handle/2099.1/19977

Montagner, L. (29 de agosto de 2018). En Puebla Capital, sobre todo en sus juntas, hay comunidades indígenas. Municipios Puebla. https://municipiospuebla.mx/ opinion/2018-08-29/en-puebla-capital-sobre-todo-ensus-juntas-hay-comunidades-ind\%C3\%ADgenas

Montesinos, M. y Sinisi, L. (2003). Pobreza, niñez y diferenciación social. Runa. Archivo para las ciencias del hombre, XXIV (1), 63-81.

Navarro, V. (15 de abril de 2019). La infancia opina, pero ¿se tiene en cuenta la participación infantil en el diseño de los espacios? Fundación Arquia Blog. https:// blogfundacion. arquia.es/2019/04/la-infancia-opinapero-se-tiene-en-cuenta-la-participacion-infantil-en-eldiseno-de-los-espacios/

Osa Menor. (10 de junio de 2020). Conversaciones ANIDAR con Santiago Atrio Cerezo. [Archivo de Vídeo]. Youtube. https://www.youtube.com/watch?v=illOypyAhzQ
Pol, E. (1996). La apropiación del espacio. En L. Iñiguez y E. Pol (Eds.), Cognición, representación y apropición del espacio (pp. 45-62). Universitat de Barcelona.

Rabello, L. (2001). Una teoría de la infancia en la contemporaneidad. En L. Rabello, Infancia y adolescencia en la cultura del consumo (pp. 21-54). Lumen.

Rasse, A. y Letelier, F. (2012). El proceso de reconstrucción de viviendas en el centro de Talca: fotografía a dos años de la catástrofe. Revista INVI, 28 (77), 139-164.

Shabel, P. (2014). Los niños y niñas como constructores de conocimiento: un caso de investigación participativa. Revista Latinoamericana de Ciencias Sociales, Niñez y Juventud, 12 (1), 159-170. http://dx.doi. org/10.11600/1692715x.1218051713

Tonucci, F. (2015). La ciudad de los niños. Grao.

Vidal, T. y Pol, E. (2005). La apropiación del espacio: una propuesta teórica para comprender la vinculación entre las personas y los lugares. Anuario de Psicología, 36 (3), 281-297.

Vidal, T., Remesar, A., Ricart, N. y Raba, A. (2008). Seis aspectos de la participación en procesos de transformación urbana. Revista de Educación Social, (7). https://eduso.net/res/revista/7/enfoques/seis-aspectosde-la-participacion-en-procesos-de-transformacionurbana 